\title{
Preface to JAISE 13(4)
}

\author{
Vincent Tam ${ }^{\mathrm{a}}$, Hamid Aghajan ${ }^{\mathrm{b}}$, Juan Carlos Augusto ${ }^{\mathrm{c}}$ and Andrés Muñoz ${ }^{\mathrm{d}}$ \\ ${ }^{a}$ Department of Electrical and Electronic Engineering, The University of Hong Kong, China \\ $\mathrm{b}^{\mathrm{b}}$ imec, IPI, Department of Telecommunications and Information Processing, Gent University, Belgium \\ ${ }^{\mathrm{c}}$ Department of Computer Science and Research Group on Development of Intelligent Environments, \\ Middlesex University, UK \\ d Polytechnic School, Universidad Católica de Murcia, Spain
}

\section{This issue}

This regular issue of JAISE is composed of four articles. The review process for the articles in this issue was supervised by our editors Carles Gomez, Francesco Furfari, and Vincent Tam, whom we thank for their service.

Many real-world applications in the fields of assisted living, Industry 4.0, smart buildings, smart cities, smart homes and intelligent robotics are fuelled by breakthroughs in computing and sensing technologies of the underlying Embedded Systems (ES) and Internet of Things (IoT) platforms. Most of the practical ES and IoT applications often involve a diversity of sensed data possibly stored in different locations to capture and predict the regular patterns of activities which occur in various smart home or office environments. Therefore, to construct a resilient computational model for capturing and predicting human activities accurately in such smart environments, the specific implementations of ambient intelligence concepts to process and analyse the sensed data is a very critical yet challenging task. The manuscript "A machine learning approach to predict the activity of smart home inhabitant" by Marufuzzaman et al. examines the structure of an intelligent network of smart home appliances with a decision tree-based machine learning approach for predicting the home inhabitants' activities through different input data types such as locations, states and time of use of various home appliances. To minimize the execution time, a new and incremental approach to generate the underlying decision tree to predict the next activity or event is adopted. The proposed machine learning algorithm attains an accuracy of $90 \%$ to predict the involved locations based on a well-known smart home dataset. In addition, a hardware prototype is successfully designed and implemented to validate the accuracy and performance of the proposed system.

Besides predicting the inhabitants' activities with accuracy in smart homes, applying ambient intelligence and smart sensors such as infrared cameras may help to provide continuous monitoring of the elderly for fall detection or other safety concerns. The manuscript entitled "Real time fall detection using infrared cameras and reflective tapes under day/night luminance" by Ramanujama and Padmavathi proposes an intelligent, real-time and 24/7 monitoring system that provides a practical solution with affordable infrared cameras for effective fall detection of the elderly even when there is change in the illumination of the underlying smart environment. In addition, through fitting an ellipse to the body of the subject in each video frame, the orientation angle of the body is measured, and a classifier is trained to detect any falls.

In addition to different smart home environments, intelligent modelling approaches can be utilized as effective assistive living technologies for smart city applications. The manuscript "An intelligent model to assist people with disabilities in smart cities" by Telles et al. proposes an effective model called MASC for assistive smart cities covering geographically broad areas such as cities and metropolitan regions while supporting a diversity of disabilities including hearing, visual impairment, and limitation of lower limb movements. Moreover, MASC uses the interactions of people with disabilities (PwD) to compose histories of contexts which it uses to offer services. 
Critical evaluation of the intelligent model is carried out based on performance, functionality and usability measures. More importantly, MASC provides services for different types of users including the PwD, healthcare professionals and public administration of assistive smart cities.

Smart buildings are surely a very significant part of any smart city application. In numerous smart buildings, the accuracy of localization methods using energy-efficient and small-sized sensors and beacons is crucial in providing appropriate services to the building occupants. The manuscript "Accuracy analysis of BLE beacon-based localization in smart buildings" by Ivanov conducts a thorough analysis of the accuracy of indoor localization using Bluetooth Low Energy (BLE) beacons. The key objective of the investigation is to present an approach creating accurate Indoor Positioning Systems (IPS) using algorithms to be implemented in a real-time manner with a relatively low computing power. Moreover, significant system parameters including the specific localization algorithm, density of the beacons, deployment strategy, and noise in the BLE channels are carefully analysed. Accordingly, an adaptive algorithm to pre-process the signals received from the beacons is proposed to reduce the level of noise while capturing the dynamics of the visitors. Experiments conducted in both simulated and real environments reveal that and accuracy of indoor localization within the range of less than one meter can be obtained through the proposed technique.

\section{Upcoming issues}

The following is a list of upcoming issues of JAISE:

- September 2021: Thematic Issue on Deep Learning-based Real-time Visual Analytics in a Smart City.

- November 2021: Regular Issue.

- January 2022: Thematic Issue on Sensing and Computing for Smart Healthcare.

- March 2022: Regular Issue.

- May 2022: Thematic Issue on Secure and Advanced Technology for Intelligent Environments.

More information on the call for papers to the future issues is available on the webpage of JAISE at: http://www. iospress.nl/journal/journal-of-ambient-intelligence-and-smart-environments/. 